\title{
The Boole polynomials associated with the $p$-adic gamma function
}

\author{
Ugur Duran and Mehmet Acikgoz
}

\begin{abstract}
The main aim of this paper is to set some correlations between Boole polynomials and $p$-adic gamma function in conjunction with $p$-adic Euler contant. We develop diverse formulas for $p$-adic gamma function by means of their Mahler expansion and fermionic $p$-adic integral on $\mathbb{Z}_{p}$. Also, we acquire two fermionic $p$-adic integrals of $p$-adic gamma function in terms of Boole numbers and polynomials. We then provide fermionic $p$-adic integral of the derivative of $p$-adic gamma function and a representation for the $p$-adic Euler constant by means of the Boole polynomials. Furthermore, we investigate an explicit representation for the aforesaid constant covering Stirling numbers of the first kind.
\end{abstract}

\section{Introduction}

Let $\mathbb{N}:=\{1,2,3, \cdots\}$ and $\mathbb{N}_{0}=\mathbb{N} \cup\{0\}$. Throughout this paper, $\mathbb{Z}$ denotes the set of integers, $\mathbb{R}$ denotes the set of real numbers and $\mathbb{C}$ denotes the set of complex numbers. Let $p$ be chosen as an odd fixed prime number. The symbols $\mathbb{Z}_{p}, \mathbb{Q}_{p}$ and $\mathbb{C}_{p}$ denote the ring of $p$-adic integers, the field of $p$-adic numbers and the completion of an algebraic closure of $\mathbb{Q}_{p}$, respectively. The normalized absolute value according to the theory of $p$-adic analysis is given by $|p|_{p}=p^{-1}$ (for details, cf. [1-12]; see also the related references cited therein).

The fermionic $p$-adic integral on $\mathbb{Z}_{p}$ of a function

$$
f \in C\left(\mathbb{Z}_{p}\right)=\left\{f \mid f: \mathbb{Z}_{p} \rightarrow \mathbb{Z}_{p} \text { be a continuous function }\right\}
$$

is defined $(c f .[5,12])$ as follows:

$$
\int_{\mathbb{Z}_{p}} f(x) d \mu_{-1}(x)=\lim _{N \rightarrow \infty} \frac{1}{p^{N}} \sum_{k=0}^{p^{N}-1}(-1)^{k} f(k) .
$$

1991 Mathematics Subject Classification. Primary 05A10, 05A30; Secondary 11B65, 11S80, 33B15.

Key words and phrases. p-adic numbers, p-adic gamma function, p-adic Euler constant, Mahler expansion, Boole polynomials, Stirling numbers of the first kind.

Communicated by ... 
By (1.1), the following integral equation holds true, see [1,2,5-7]:

$$
\int_{\mathbb{Z}_{p}} f(x+1) d \mu_{-1}(x)+\int_{\mathbb{Z}_{p}} f(x) d \mu_{-1}(x)=2 f(0),
$$

which intensely holds usability in introducing assorted generalizations of many special polynomials such as Euler, Genocchi, Frobenius-Euler and Changhee polynomials, see $[1,2,4-7,12]$.

The familiar Boole polynomials $B l_{n}(x)$ of the first kind are defined by means of the following generating function $(c f .[7])$ :

$$
\sum_{n=0}^{\infty} B l_{n}(x \mid \omega) \frac{t^{n}}{n !}=\frac{1}{1+(1+t)^{\omega}}(1+t)^{x}=\int_{\mathbb{Z}_{p}}(1+t)^{x+\omega y} d \mu_{-1}(y) .
$$

When $\omega=1$, we have $B l_{n}(x \mid 1):=2^{-1} C h_{n}(x)$ which are the Changhee polynomials given by the following generating function to be $(c f .[6])$

$$
\sum_{n=0}^{\infty} C h_{n}(x) \frac{t^{n}}{n !}=\frac{2}{2+t}(1+t)^{x}
$$

In the case $x=0$ in the (1.4), one can get $C h_{n}(0):=C h_{n}$ standing for $n$-th Changhee number $(c f .[3,8])$.

The Boole polynomials of the first kind can be represented by

$$
B l_{n}(x \mid \omega)=2^{-1} \int_{\mathbb{Z}_{p}}(x+\omega y)_{n} d \mu_{-1}(y),
$$

where $(x)_{n}$ be falling factorial given by $(c f .[1-3,8,9])$

$$
(x)_{n}=x(x-1)(x-2) \cdots(x-n+1) .
$$

In the special case, $B l_{n}(0 \mid \omega):=B l_{n}(\omega)$ is called $n$-th Boole number.

The Boole polynomials of the second kind are defined by means of the following fermionic $p$-adic integral, see [6]:

$$
\sum_{n=0}^{\infty} \widehat{B l}_{n}(x \mid \omega) \frac{t^{n}}{n !}=\frac{1}{2} \int_{\mathbb{Z}_{p}}(1+t)^{x-\omega y} d \mu_{-1}(y)=\frac{(1+t)^{\omega}}{1+(1+t)^{\omega}}(1+t)^{x},
$$

which also means

$$
\widehat{B l}_{n}(x \mid \omega)=2^{-1} \int_{\mathbb{Z}_{p}}(x-\omega y)_{n} d \mu_{-1}(y) .
$$

When $x=0$, we have $\widehat{B l}_{n}(0 \mid \omega):=\widehat{B l}_{n}(\omega)$ which is called the Boole numbers of the second kind, $c f$. [6].

In recent years, the Boole and the Changhee polynomials with their several generalizations studied and developed by a lot of mathematicians possess various applications in $p$-adic analysis, see $[2,4,6,7]$ and also references cited therein.

The formula (1.6) satisfies the following identity:

$$
(x)_{n}=\sum_{k=0}^{n} S_{1}(n, k) x^{k},
$$


where $S_{1}(n, k)$ denotes the Stirling numbers of the first kind (cf. $\left.[1,2,4,6,7]\right)$.

The following relation holds true for $n \geqslant 0$ :

$$
\int_{\mathbb{Z}_{p}}\left(\begin{array}{c}
x+\omega y \\
n
\end{array}\right) d \mu_{-1}(y)=\sum_{m=0}^{n} \omega^{m} S_{1}(n, m) E_{m}\left(\frac{x}{\omega}\right),
$$

where $E_{m}(x / \omega)$ denotes $m$-th Euler polynomials with the value $x / \omega$ defined by ( $c f$. $[6])$

$$
\sum_{n=0}^{\infty} E_{n}(y) \frac{t^{n}}{n !}=\int_{\mathbb{Z}_{p}}(x+y)^{n} d \mu_{-1}(x)=\frac{2}{e^{t}+1} e^{y t} .
$$

Note that when $y=0$, we have $E_{n}(0):=E_{n}$ called $n$-th Euler number (see $[\mathbf{6}]$ ).

In this paper, we investigate several relations for $p$-adic gamma function by means of their Mahler expansion and fermionic $p$-adic integral on $\mathbb{Z}_{p}$. We also derived two fermionic $p$-adic integrals of $p$-adic gamma function in terms of Boole polynomials and numbers. Moreover, we discover fermionic $p$-adic integral of the derivative of $p$-adic gamma function. We acquire a representation for the $p$-adic Euler constant by means of the Boole polynomials. We finally develop a novel, explicit and interesting representation for the $p$-adic Euler constant covering Stirling numbers of the first kind.

\section{The Boole polynomials related to $p$-adic gamma function}

Throughout this paper, we suppose that $t \in \mathbb{C}_{p}$ with $|t|_{p}<p^{-\frac{1}{1-p}}$. In this part, we perform to derive some relationships among the two types of Boole polynomials, $p$-adic gamma function and $p$-adic Euler constant by making use of the Mahler expansion of the $p$-adic gamma function.

The $p$-adic gamma function (see $[3,4,8-11]$ ) is given by

$$
\Gamma_{p}(x)=\lim _{n \rightarrow x}(-1)^{n} \prod_{\substack{j<n \\(p, j)=1}} j \quad\left(x \in \mathbb{Z}_{p}\right),
$$

where $n$ approaches $x$ through positive integers.

The $p$-adic Euler constant $\gamma_{p}$ is given by

$$
\gamma_{p}:=-\frac{\Gamma_{p}^{\prime}(1)}{\Gamma_{p}(0)}=\Gamma_{p}^{\prime}(1)=-\Gamma_{p}^{\prime}(0) .
$$

The $p$-adic gamma function in conjunction with its various generalizations and $p$ adic Euler constant have been investigated and studied by many mathematicians, $c f$. [3, 4, 8-11]; see also the references cited in each of these earlier works.

For $x \in \mathbb{Z}_{p}$, the symbol $\left(\begin{array}{l}x \\ n\end{array}\right)$ is given by

$$
\left(\begin{array}{l}
x \\
0
\end{array}\right)=1 \text { and }\left(\begin{array}{l}
x \\
n
\end{array}\right)=\frac{x(x-1) \cdots(x-n+1)}{n !} \quad(n \in \mathbb{N}) .
$$

Let $x \in \mathbb{Z}_{p}$ and $n \in \mathbb{N}$. The functions $x \rightarrow\left(\begin{array}{l}x \\ n\end{array}\right)$ form an orthonormal base of the space $C\left(\mathbb{Z}_{p} \rightarrow \mathbb{C}_{p}\right)$ with respect to the Euclidean norm $\|\cdot\|_{\infty}$. The mentioned 
orthonormal base satisfy the formula below:

$$
\left(\begin{array}{l}
x \\
n
\end{array}\right)^{\prime}=\sum_{j=0}^{n-1} \frac{(-1)^{n-j-1}}{n-j}\left(\begin{array}{l}
x \\
j
\end{array}\right) \quad(\text { see }[\mathbf{9}] \text { and }[\mathbf{1 1}]) .
$$

Kurt Mahler, German mathematician, provided an extension for continuous maps of a $p$-adic variable using the special polynomials as binomial coefficient polynomial [9] in 1958 as follows. form

Theorem 1. [9] Every continuous function $f: \mathbb{Z}_{p} \rightarrow \mathbb{C}_{p}$ can be written in the

$$
f(x)=\sum_{n=0}^{\infty} a_{n}\left(\begin{array}{l}
x \\
n
\end{array}\right)
$$

for all $x \in \mathbb{Z}_{p}$, where $a_{n} \in \mathbb{C}_{p}$ and $a_{n} \rightarrow 0$ as $n \rightarrow \infty$.

The base $\left\{\left(\begin{array}{l}* \\ n\end{array}\right): n \in \mathbb{N}\right\}$ is termed as Mahler base of the space $C\left(\mathbb{Z}_{p} \rightarrow \mathbb{C}_{p}\right)$, and the components $\left\{a_{n}: n \in \mathbb{N}\right\}$ in $f(x)=\sum_{n=0}^{\infty} a_{n}\left(\begin{array}{l}x \\ n\end{array}\right)$ are called Mahler coefficients of $f \in C\left(\mathbb{Z}_{p} \rightarrow \mathbb{C}_{p}\right)$. The Mahler expansion of the $p$-adic gamma function $\Gamma_{p}$ and its Mahler coefficients are given in [11] as follows.

Proposition 2. For $x \in \mathbb{Z}_{p}$, let $\Gamma_{p}(x+1)=\sum_{n=0}^{\infty} a_{n}\left(\begin{array}{l}x \\ n\end{array}\right)$ be Mahler series of $\Gamma_{p}$. Then its coefficients satisfy the following expression:

$$
\sum_{n \geqq 0}(-1)^{n+1} a_{n} \frac{x^{n}}{n !}=\frac{1-x^{p}}{1-x} \exp \left(x+\frac{x^{p}}{p}\right) .
$$

The fermionic $p$-adic integral on $\mathbb{Z}_{p}$ of the $p$-adic gamma function via Eq. (1.5) and Proposition 2 is as follows.

Theorem 3. The following identity holds true for $n \in \mathbb{N}$ :

$$
\int_{\mathbb{Z}_{p}} \Gamma_{p}(\omega x+1) d \mu_{-1}(x)=2 \sum_{n=0}^{\infty} \frac{a_{n}}{n !} B l_{n}(\omega),
$$

where $a_{n}$ is given by Proposition 2 .

Proof. For $x, \omega \in \mathbb{Z}_{p}$, by Proposition 2, we get

$$
\int_{\mathbb{Z}_{p}} \Gamma_{p}(\omega x+1) d \mu_{-1}(x)=\sum_{n=0}^{\infty} a_{n} \int_{\mathbb{Z}_{p}}\left(\begin{array}{c}
\omega x \\
n
\end{array}\right) d \mu_{-1}(x)
$$

and using the formula (1.5), we acquire

$$
\int_{\mathbb{Z}_{p}} \Gamma_{p}(\omega x+1) d \mu_{-1}(x)=\sum_{n=0}^{\infty} \frac{2 a_{n}}{n !} B l_{n, 1}(\omega),
$$

which gives the asserted result.

We here present one other fermionic $p$-adic integral of the $p$-adic gamma function related to the Boole polynomials as follows. 
THE BOOLE POLYNOMIALS ASSOCIATED WITH THE $p$-ADIC GAMMA FUNCTION 5

Theorem 4. Let $x, y, \omega \in \mathbb{Z}_{p}$. We have

$$
\int_{\mathbb{Z}_{p}} \Gamma_{p}(x+\omega y+1) d \mu_{-1}(y)=2 \sum_{n=0}^{\infty} \frac{a_{n}}{n !} B l_{n}(x \mid \omega),
$$

where $a_{n}$ is given by Proposition 2 . we get

Proof. For $x, y, \omega \in \mathbb{Z}_{p}$, by the relation $\left(\begin{array}{c}x+\omega y \\ n\end{array}\right)=\frac{(x+\omega y)_{n}}{n !}$ and Proposition 2 ,

$$
\begin{gathered}
\int_{\mathbb{Z}_{p}} \Gamma_{p}(x+\omega y+1) d \mu_{-1}(y)=\int_{\mathbb{Z}_{p}} \sum_{n=0}^{\infty} a_{n} \frac{(x+\omega y)_{n}}{n !} d \mu_{-1}(y) \\
=\sum_{n=0}^{\infty} a_{n} \frac{1}{n !} \int_{\mathbb{Z}_{p}}(x+\omega y)_{n} d \mu_{-1}(y),
\end{gathered}
$$

which is the desired result (2.6) via (1.3).

A relation between $\Gamma_{p}(x)$ and $\widehat{B l}_{n}(x \mid \omega)$ is stated by the following theorem.

Theorem 5 . For $x, y, \omega \in \mathbb{Z}_{p}$, we have

$$
\int_{\mathbb{Z}_{p}} \Gamma_{p}(x-\omega y+1) d \mu_{-1}(y)=2 \sum_{n=0}^{\infty} a_{n} \frac{\widehat{B l}_{n}(x \mid \omega)}{n !},
$$

where $a_{n}$ is given by Proposition 2 . we get

Proof. For $x, y, \omega \in \mathbb{Z}_{p}$, by the relation $\left(\begin{array}{c}x-\omega y \\ n\end{array}\right)=\frac{(x-\omega y)_{n}}{n !}$ and Proposition 2 ,

$$
\begin{gathered}
\int_{\mathbb{Z}_{p}} \Gamma_{p}(x-\omega y+1) d \mu_{-1}(y)=\int_{\mathbb{Z}_{p}} \sum_{n=0}^{\infty} a_{n} \frac{(x-\omega y)_{n}}{n !} d \mu_{-1}(y) \\
=\sum_{n=0}^{\infty} a_{n} \frac{1}{n !} \int_{\mathbb{Z}_{p}}(x-\omega y)_{n} d \mu_{-1}(y),
\end{gathered}
$$

which is the desired result thanks to (1.8).

A consequence of Theorem 5 is given by the following corollary.

Corollary 6 . Upon setting $x=0$ in Theorem 5 gives the following relation for $\Gamma_{p}$ and $\widehat{B l}_{n}(\omega)$ :

$$
\int_{\mathbb{Z}_{p}} \Gamma_{p}(-\omega y+1) d \mu_{-1}(y)=2 \sum_{n=0}^{\infty} a_{n} \frac{\widehat{B l}_{n}(\omega)}{n !},
$$

where $a_{n}$ is given by Proposition 2 .

Here is the fermionic $p$-adic integral of the derivative of the $p$-adic gamma function. 
Theorem 7 . For $x, y, \omega \in \mathbb{Z}_{p}$, we have

$$
\int_{\mathbb{Z}_{p}} \Gamma_{p}^{\prime}(x+\omega y+1) d \mu_{-1}(y)=2 \sum_{n=0}^{\infty} \sum_{j=0}^{n-1} a_{n} \frac{(-1)^{n-j-1} B l_{j}(x \mid \omega)}{(n-j) j !} .
$$

Proof. In view of Proposition 2, we obtain

$$
\begin{gathered}
\int_{\mathbb{Z}_{p}} \Gamma_{p}^{\prime}(x+\omega y+1) d \mu_{-1}(y)=\int_{\mathbb{Z}_{p}} \sum_{n=0}^{\infty} a_{n}\left(\begin{array}{c}
x+\omega y \\
n
\end{array}\right)^{\prime} d \mu_{-1}(y) \\
=\sum_{n=0}^{\infty} a_{n} \int_{\mathbb{Z}_{p}}\left(\begin{array}{c}
x+\omega y \\
n
\end{array}\right)^{\prime} d \mu_{-1}(y)
\end{gathered}
$$

and using (2.3), we derive

$$
\begin{aligned}
\int_{\mathbb{Z}_{p}} \Gamma_{p}^{\prime}(x+\omega y+1) d \mu_{-1}(y) & =\sum_{n=0}^{\infty} \sum_{j=0}^{n-1} a_{n} \frac{(-1)^{n-j-1}}{n-j} \int_{\mathbb{Z}_{p}}\left(\begin{array}{c}
x+\omega y \\
j
\end{array}\right) d \mu_{-1}(y) \\
& =2 \sum_{n=0}^{\infty} \sum_{j=0}^{n-1} a_{n} \frac{(-1)^{n-j-1}}{n-j} \frac{B l_{j}(x \mid \omega)}{j !} .
\end{aligned}
$$

The immediate result of Theorem 7 is given as follows.

Corollary 8. For $y \in \mathbb{Z}_{p}$, we have

$$
\int_{\mathbb{Z}_{p}} \Gamma_{p}^{\prime}(\omega y+1) d \mu_{-1}(y)=2 \sum_{n=0}^{\infty} \sum_{j=0}^{n-1} a_{n} \frac{(-1)^{n-j-1} B l_{j}(\omega)}{(n-j) j !} .
$$

We now provide a new and interesting representation of the $p$-adic Euler constant by means of Boole polynomials of the second kind.

TheOREM 9. We have

$$
\gamma_{p}=\sum_{n=0}^{\infty} \sum_{j=0}^{n-1} a_{n}(-1)^{n-j} \frac{B l_{j}(\omega-1 \mid \omega)-B l_{j}(-1 \mid \omega)}{(n-j) j !} .
$$

Proof. Taking $f(y)=\Gamma_{p}^{\prime}(\omega y)$ in (1.2) yields the following result

$$
\int_{\mathbb{Z}_{p}} \Gamma_{p}^{\prime}(\omega y+\omega-1+1) d \mu_{-1}(y)+\int_{\mathbb{Z}_{p}} \Gamma_{p}^{\prime}(\omega y) d \mu_{-1}(y)=2 \Gamma_{p}^{\prime}(0) .
$$

Using (2.2), (2.7) and Theorem 7 along with some basic calculations, we have $2 \sum_{n=0}^{\infty} \sum_{j=0}^{n-1} a_{n} \frac{(-1)^{n-j-1} B l_{j}(\omega-1 \mid \omega)}{(n-j) j !}+2 \sum_{n=0}^{\infty} \sum_{j=0}^{n-1} a_{n} \frac{(-1)^{n-j-1} B l_{j}(-1 \mid \omega)}{(n-j) j !}=-2 \gamma_{p}$ which implies the asserted result.

We give the following explicit formula for the $p$-adic Euler constant. 
THE BoOle POLYNomials ASSOCIATED With THE $p$-ADIC GAMma FUnCtion 7

TheOREM 10. The following explicit formula is valid:

$$
\begin{aligned}
\gamma_{p}= & \sum_{n=0}^{\infty} \sum_{j=0}^{n-1} \frac{a_{n}}{(n-j) j !} \sum_{m=0}^{\infty}(-1)^{m+n-j} \\
& \cdot \sum_{k=0}^{n} S_{1}(n, k)\left((-1-\omega m)^{k}-(-1-\omega-\omega m)^{k}\right) .
\end{aligned}
$$

Proof. By (1.7), we get

$$
\begin{aligned}
\sum_{n=0}^{\infty} \widehat{B l}_{n}(x \mid \omega) \frac{t^{n}}{n !} & =\frac{1}{1+(1+t)^{\omega}}(1+t)^{x+\omega}=\sum_{m=0}^{\infty}(-1)^{m}(1+t)^{x+\omega+\omega m} \\
& =\sum_{m=0}^{\infty}(-1)^{m}(1+t)^{x+\omega+\omega m}=\sum_{m=0}^{\infty}(-1)^{m} \sum_{n=0}^{\infty}\left(\begin{array}{c}
x+\omega+\omega m \\
n
\end{array}\right) t^{n} \\
& =\sum_{n=0}^{\infty}\left(\sum_{m=0}^{\infty}(-1)^{m}(x+\omega+\omega m)_{n}\right) \frac{t^{n}}{n !}
\end{aligned}
$$

which gives, from (1.9), that

$$
\widehat{B l}_{n}(x \mid \omega)=\sum_{m=0}^{\infty}(-1)^{m} \sum_{k=0}^{n} S_{1}(n, k)(x+\omega+\omega m)^{k} .
$$

In view of (1.5) and (1.8), we easily obtain that

$$
\widehat{B l}_{n}(x \mid \omega)=B l_{n}(x \mid-\omega) .
$$

So, we derive that

$$
B l_{n}(x \mid \omega)=\sum_{m=0}^{\infty}(-1)^{m} \sum_{k=0}^{n} S_{1}(n, k)(x-\omega-\omega m)^{k} .
$$

Thus, we have

$$
B l_{n}(-1 \mid \omega)=\sum_{m=0}^{\infty}(-1)^{m} \sum_{k=0}^{n} S_{1}(n, k)(-1-\omega-\omega m)^{k}
$$

and

$$
B l_{n}(\omega-1 \mid \omega)=\sum_{m=0}^{\infty}(-1)^{m} \sum_{k=0}^{n} S_{1}(n, k)(-1-\omega m)^{k} .
$$

By combining (2.8), (2.10) and (2.11), we arrive at the desired result.

\section{Conclusions and Observations}

In this work, we first have handled some multifarious relations for the $p$-adic gamma function and the Boole polynomials of both sides. We also have acquired the fermionic $p$-adic integral of the derivative of $p$-adic gamma function. We then have obtained a new representation for the $p$-adic Euler constant via the Boole 
polynomials of both kinds. Lastly, we have investigated an interesting identity for the mentioned constant.

Authors' Note. The authors declare that the paper is an original work, submitted only to this journal, have not been published previously whole or in the part, and will not be submitted elsewhere until final decision of the Editors in Publications de l'Institut Mathematique.

\section{References}

[1] U. Duran, M. Acikgoz, On $(\rho, q)$-Euler numbers and polynomials associated with $(\rho, q)$ Volkenborn integrals, Int. J. Number Theory, 14 (1), 2018, 241-253.

[2] F. G. Gurkan, M. Acikgoz, E. Agyuz, A study on the new mixed-type polynomials related to Boole polynomials, Afr. Mat., 28, 2017, 279-290.

[3] Ö. Ç. Havare, H. Menken, The Volkenborn integral of the p-adic gamma function, Int. J. Adv. Appl. Funct., 5 (2), 2018, 56-59.

[4] Ö. Ç. Havare, H. Menken, A note on p-adic gamma function and q-Changhee polynomials, J. Math. Comput. Sci., 18, 2018, 11-17.

[5] T. Kim, A note on p-adic invariant integral in the rings of p-adic integers, Adv. Stud. Contemp. Math., 13 (1), 2006, 95-99.

[6] D. S. Kim, T. Kim, A note on Boole polynomials, Integral Transforms Spec. Funct., 25 (8), 2014, 627-633.

[7] T. Kim, D. S. Kim, Barnes-type Boole polynomials, Contrib. Discrete Math., 11 (1), 2016, $7-15$.

[8] Y. S. Kim, q-analogues of p-adic gamma functions and p-adic Euler constants, Comm. Korean Math. Soc., 13 (4), 1998, 735-741.

[9] K. Mahler, An interpolation series for continuous functions of a p-adic variable, J. Reine Angew. Math., 199, 1958, 23-34.

[10] Y. Morita, A p-adic analogue of the $\Gamma$-function, J. Fac. Sci., 22 (2), 1975, 255-266.

[11] A. M. Robert, A course in p-adic analysis, Springer-Verlag New York, Inc., 2000.

[12] K. Shiratani, S. Yamamoto, On a p-adic interpolation function for Euler numbers and its derivatives, Mem. Fac. Sci., Kyushu Univ. Ser. A, 39 (1), 1985, 113-125.

E-mail address: mtdrnugur@gmail.com - acikgoz@gantep.edu.tr

Department of Basic Sciences of Engineering, İskenderun Tecnical University, HATAY, TURKEY

Department of Mathematics, University of Gaziantep, Gaziantep, Turkey 